\title{
Isolated Distal Deep Vein Thrombosis: Perspectives from the GARFIELD-VTE Registry
}

\author{
Sebastian M. Schellong ${ }^{1}$ Samuel Z. Goldhaber ${ }^{2}$ Jeffrey I. Weitz ${ }^{3}$ Walter Ageno ${ }^{4}$ Henri Bounameaux ${ }^{5}$
} Alexander G. G. Turpie ${ }^{6}$ Pantep Angchaisuksiri ${ }^{7}$ Sylvia Haas ${ }^{8}$ Shinya Goto ${ }^{9}$ Audrey Zaghdoun ${ }^{10}$ Alfredo Farjat ${ }^{10}$ Joern Dalsgaard Nielsen ${ }^{11}$ Gloria Kayani ${ }^{10}$ Lorenzo G. Mantovani ${ }^{12}$

Paolo Prandoni ${ }^{13}$ Ajay K. Kakkar ${ }^{14}$

${ }^{1}$ Medical Division 2, Municipal Hospital Dresden-Friedrichstadt, Dresden, Germany

2 Harvard Medical School, Harvard University, Boston, Massachusetts, United States

${ }^{3}$ Thrombosis and Atherosclerosis Research Institute, McMaster University, Hamilton, Ontario, Canada

${ }^{4}$ Department of Medicine and Surgery, University of Insubria, Varese, Italy

${ }^{5}$ Faculty of Medicine, University Hospitals of Geneva, Geneva, Switzerland

${ }^{6}$ McMaster University, Hamilton, Ontario, Canada

7 Department of Medicine, Ramathibodi Hospital, Mahidol University, Bangkok, Thailand

${ }^{8}$ Department of Medicine, Technical University of Munich, Munich, Germany

${ }^{9}$ Department of Medicine (Cardiology), Tokai University School of Medicine, Tokyo, Japan

Thromb Haemost 2019;119:1675-1685.
Address for correspondence Sebastian M. Schellong, MD, Medical Department 2, Municipal Hospital Dresden, Friedrichstraße 41, 01067 Dresden, Germany

(e-mail: sebastian.schellong@klinikum-dresden.de).

\footnotetext{
${ }^{10}$ Thrombosis Research Institute, London, United Kingdom

${ }^{11}$ Copenhagen University Hospital, Copenhagen, Denmark

${ }^{12}$ University Degli Studi di Milano Bicocca, Milan, Italy

${ }^{13}$ Arianna Foundation on Anticoagulation, Bologna, Italy

${ }^{14}$ Thrombosis Research Institute, University College London, London, United Kingdom
}

\section{Abstract}

\section{Keywords}

- deep vein thrombosis

- isolated distal deep vein thrombosis

- 12-month observational study

- GARFIELD-VTE registry
Isolated distal deep vein thrombosis (IDDVT) represents up to half of all lower limb DVT. This study investigated treatment patterns and outcomes in 2,145 patients with IDDVT in comparison with those with proximal DVT (PDVT; $n=3,846$ ) and pulmonary embolism (PE; $n=4,097$ ) enrolled in the GARFIELD-VTE registry. IDDVT patients were more likely to have recently undergone surgery $(14.6 \%)$ or experienced leg trauma $(13.2 \%)$ than PDVT patients (11.0 and $8.7 \%$, respectively) and PE patients (12.7 and $4.5 \%$, respectively). Compared with IDDVT, patients with PDVT or PE were more likely to have active cancer (7.2\% vs. 9.9\% and 10.3\%). However, influence of provoking factors on risk of recurrence in IDDVT remains controversial. Nearly all patients (IDDVT, PDVT, and PE) were given anticoagulant therapy. In IDDVT, PDVT, and PE groups the proportion of patients receiving anticoagulant therapy was $61.4,73.9$, and $81.1 \%$ at 6 months and $45.8,54.7$, and $61.9 \%$ at 12 months. Over 12 months, the incidence of all-cause mortality, cancer, and recurrence was significantly lower in IDDVT patients than PDVT patients (hazard ratio [HR], 0.61 [95\% confidence interval [Cl], 0.48-0.77]; sub-HR [sHR], 0.60 [95\% Cl, 0.39-0.93]; and sHR, 0.76 [95\% Cl, 0.60-0.97]). Likewise, risk of death and incident cancer was significantly (both $p<0.05$ ) lower in patients with IDDVT compared with PE. This study reveals a global trend that most IDDVT patients as well as those with PDVT and PE are given anticoagulant therapy, in many cases for at least 12 months. received

January 3, 2019

accepted after revision May 29, 2019
DOI https://doi.org/ 10.1055/s-0039-1693461. ISSN 0340-6245.
(C) 2019 Georg Thieme Verlag KG Stuttgart · New York
License terms

()(1) $\odot \circledast$ 


\section{Introduction}

Venous thromboembolism (VTE) encompasses deep vein thrombosis (DVT) and pulmonary embolism (PE). ${ }^{1,2}$ According to the International Society on Thrombosis and Haemostasis (ISTH), VTE affects 115 to 269 persons per 100,000 population worldwide. ${ }^{3,4}$ In the United States, mortality due to VTE has been estimated in the range of 9.4 to 32.3 per $100,000 .^{3}$ DVT affecting the lower limbs can be broadly divided anatomically into proximal (PDVT) and distal DVT. PDVT involves the iliac, femoral, or popliteal veins. Distal DVT is located below the level of the knee joint involving the posterior and anterior tibial, peroneal, and calf muscle veins. ${ }^{5}$ DVT detectable only in the distal leg veins-isolated distal DVT (IDDVT)-represents 20 to $50 \%$ of all lower limb DVT. ${ }^{6}$

The optimal management of IDDVT is not well defined. ${ }^{7-9}$ Most DVT arises in the calf veins, and although many cases spontaneously resolve, some may propagate to proximal leg veins, typically within a short space of time. ${ }^{5,10}$ Proximal thrombi are more likely to embolize than distal thrombi. ${ }^{5}$ Studies investigating the proportion of untreated/treated IDDVT patients who are at risk of proximal extension have produced wide-ranging results. ${ }^{5,6,11}$ Of note, a recent randomized controlled trial of anticoagulant therapy in low-risk patients with IDDVT did not demonstrate any reduction of adverse outcomes compared with no treatment, albeit the trial was stopped prematurely due to slow recruitment. ${ }^{12}$

International treatment guidelines such as those issued by the American College of Chest Physicians (ACCP) ${ }^{13,14}$ recommend giving anticoagulant therapy in patients with PDVT or PE as well as IDDVT with severe symptoms or risk factors for extension to proximal veins (inpatients, prior history of VTE, cancer). Optimal duration of anticoagulant therapy is based on whether DVT is provoked, unprovoked (idiopathic), or associated with cancer. Surveillance using compression ultrasonography (CUS) over 2 weeks without initiating anticoagulation is suggested in low-risk IDDVT patients. However, these are "weak" recommendations based on low-quality evidence (grade $2 \mathrm{C}$ ). ${ }^{14}$ Moreover, as the ACCP guidelines acknowledge, in the absence of long-term safetymonitoring studies the relative usefulness of newer "direct oral anticoagulant" (DOAC) therapies against VTE remains unclear. $^{14,15}$

This study investigated the clinical characteristics, treatment patterns, and 1-year outcomes in patients with IDDVT in comparison with those with PDVT ( \pm distal) and PE ( \pm any DVT) enrolled in the GARFIELD-VTE registry.

\section{Methods}

\section{Study Design and Participants}

The GARFIELD-VTE registry (ClinicalTrials.gov identifier: NCT02155491) is an ongoing, prospective, noninterventional, observational study of 10,685 patients with objectively diagnosed VTE, from 415 sites in 28 countries. The study design has been previously reported. ${ }^{16}$ Patients were recruited from centers representative of the various care settings for each country. They were consecutively enrolled, and unselected. No specific treatments, tests, or procedures were mandated by the study protocol. Decisions to initiate, continue, or change treatment were solely at the discretion of treating physicians.

The registry records treatment patterns for acute VTE as well as the rate and nature of VTE recurrence, bleeding complications, and all-cause mortality over 36 months of follow-up. Patients were recruited across a range of clinical settings, including vascular medicine, general practice, and internal medicine. Patients aged $\geq 18$ years with a confirmed diagnosis of VTE within 30 days of entry into the registry were eligible for inclusion. Excluded were patients with superficial vein thrombosis, those who had not completed treatment for a previous VTE, patients participating in another interventional study, and those in whom long-term follow-up was not envisaged.

\section{Data Collection}

Data were captured by electronic case report form (eCRF), submitted to the registry-coordinating center (eClinicalHealth Services, Stirling, United Kingdom) via secure Web sites, and analyzed by the Thrombosis Research Institute, London, United Kingdom. The completeness and accuracy of data collected from medical records are checked by the registry-coordinating center and the source data verified in $10 \%$ of all cases. Data on outcomes relevant to the registry are collected through review of clinical records and patient notes. These include: patient demographics, medical history, provoking VTE risk factors (within the previous 3 months), symptoms, nature of VTE (extent and location), and method and date of diagnosis. Importantly for the present analysis, no formal definitions for IDDVT, PDVT, or PE are included in eCRFs; they include a field "type of lower limb DVT" with three options: (1) distal DVT, (2) PDVT, or (3) both distal DVT and PDVT. Routinely performed tests are documented (including hemoglobin, platelet count, international normalized ratio, and creatinine). All patients are followed prospectively for a minimum of 36 months.

\section{Outcomes}

In this real-world observational study, outcomes were recorded in standardized eCRFs at each participating centre. Outcomes of interest were incidence of recurrent VTE episode, all-cause mortality, bleeds (any or major as defined by the ISTH criteria ${ }^{17}$ ), cancer (diagnosed at least 30 days after index VTE), myocardial infarction/acute coronary syndrome, and stroke/transient ischemic attack (TIA). This study did not capture event rates according to treatment status. Outcomes were not independently adjudicated.

\section{Subanalysis: Influence of Provoking Factors on Future Risk}

A subanalysis was performed to investigate whether site of DVT interacts with known VTE provoking factors to influence risk of adverse outcomes. Patients with IDDVT and PDVT were: (1) stratified by the presence of recent, ISTH-defined ${ }^{18}$ transient $^{-}$ provoking factors (at least one of surgery, hospitalization, pregnancy, hormone replacement therapy/oral contraception, acute medical illness, or trauma of lower limb within 3 months prior to enrolment), persistent provoking factor such as active 
cancer at baseline, or none of these provoking factors; and (2) their 1-year incidence of all-cause mortality, VTE recurrence, and major bleeding was investigated.

\section{Ethics}

The registry is being conducted in accordance with the Declaration of Helsinki and guidelines from the International Conference on Harmonization on Good Clinical and Pharmacoepidemiological Practice, and adheres to all applicable national laws and regulations. Independent ethics committee for each participating country and hospital-based institutional review boards approved the registry design. All patients provided written informed consent to participate.

\section{Statistical Analyses}

Baseline demographics, regional characteristics, and treatments are presented by VTE subgroup. Continuous variables are summarized as means (standard deviation [SD]), medians (interquartile range, and ranges (min-max). Categorical factors are reported as frequency count (percentage). Event rates (per 100 person-years) and associated standard errors were estimated using a log-linked generalized linear model (Poisson regression). Large-sample 95\% confidence intervals ( $95 \%$ CIs) were obtained by inverting the link function. In this analysis, mortality was posed as a competing risk for all other clinical outcomes. Thereby, hazard ratio (HR) for death was estimated by Cox model, and sub-HRs (sHRs) for other outcomes were calculated by Fine-Gray model. ${ }^{19} \mathrm{HR}$ and sHRs were obtained adjusting for covariates sex and age. Model coefficients were assessed by Wald chi-squared test. Mean and variance of ratios for regional characteristics were estimated assuming normality and using a first-order Taylor approximation. Variance components were estimated by bootstrap method assuming a constant coefficient of variation across regions. A $p$-value of $\leq 0.05$ was considered statistically significant. Statistical analyses were performed on data extracted from the registry in May 2018, using SAS Enterprise Guide version 7.1 (SAS Institute Inc., Cary, North Carolina, United States).

\section{Results}

\section{Demographics and Pathways of Care}

Baseline clinical characteristics of the GARFIELD-VTE cohort have been reported elsewhere. ${ }^{20}$ Of the 10,088 eligible patients, 2,145 (21.3\%) were classified as having IDDVT, 3,846 (38.1\%) PDVT, and 4,097 (40.6\%) PE (see group assignation decision tree; - Fig. 1). Mean (SD) age and body mass index were $58.6(16.9)$ years and $28.4(6.6) \mathrm{kg} / \mathrm{m}^{2}$; no notable difference in these parameters was observed among the clinical subgroups (-Table 1). The majority of patients were recruited from European countries (56.2\%) and Asia (17.1\%). Individual country data on patients with a diagnosis

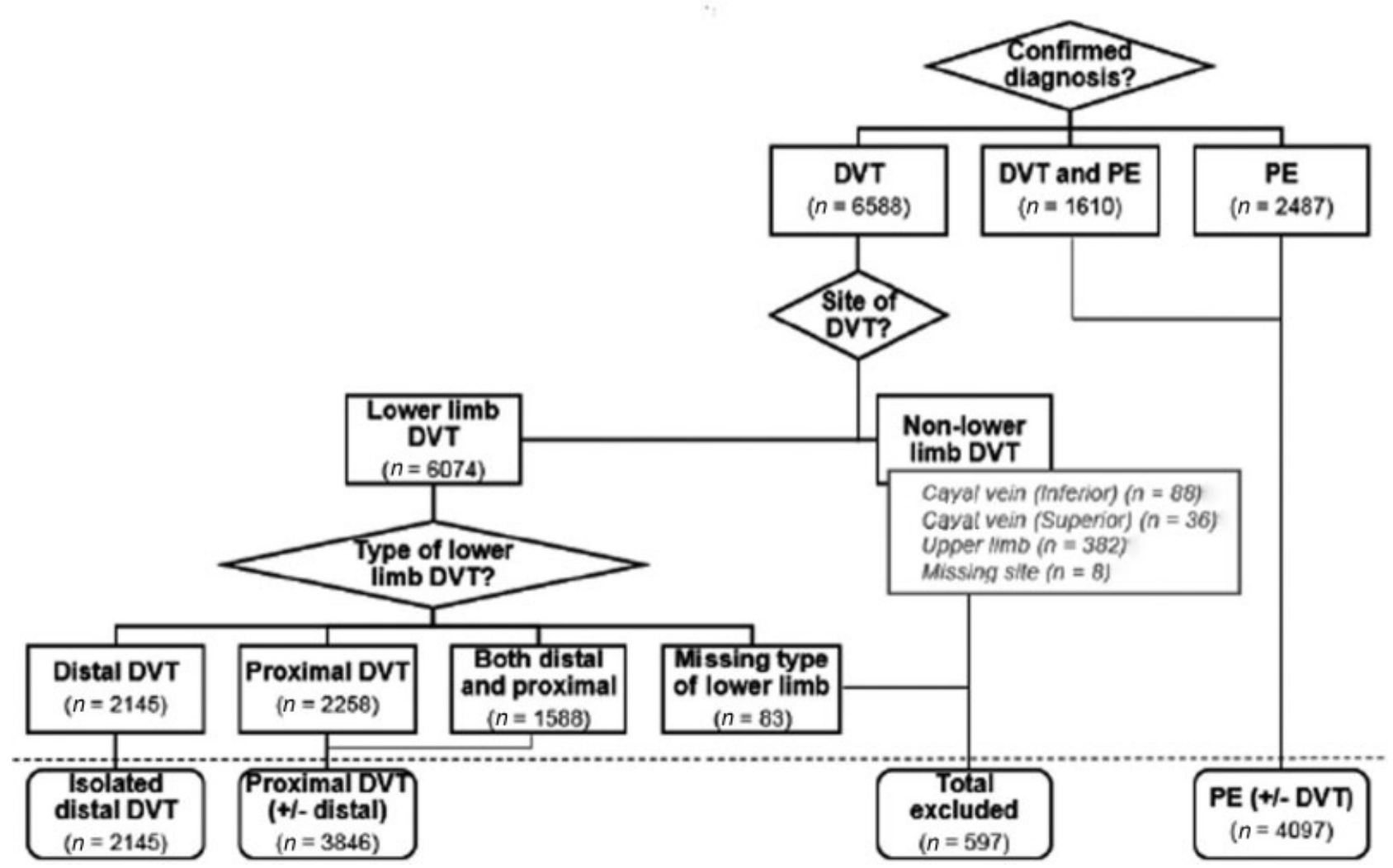

Fig. 1 Group assignation decision tree (among eligible patients). Patients with a confirmed diagnosis of pulmonary embolism (PE) irrespective of whether they had concurrent deep vein thrombosis (DVT) were assigned PE $( \pm D V T)$ group. Patients with a confirmed diagnosis of DVT without documentary evidence of PE were excluded if they presented with nonlower limb DVT; those with lower limb DVT were further dichotomized as having isolated distal DVT (IDDVT) or proximal DVT (PDVT) ( \pm distal DVT). 
Table 1 Patients' baseline demographic characteristics and care pathways

\begin{tabular}{|c|c|c|c|c|}
\hline & $\begin{array}{l}\text { IDDVT } \\
(N=2,145)\end{array}$ & $\begin{array}{l}\text { PDVT } \\
(N=3,846)\end{array}$ & $\begin{array}{l}\mathrm{PE} \\
(N=4,097)\end{array}$ & $\begin{array}{l}\text { Total } \\
(N=10,088)\end{array}$ \\
\hline \multicolumn{5}{|l|}{ Sex, $n(\%)$} \\
\hline Male & $1,029(48.0)$ & $1,958(50.9)$ & $2,079(50.7)$ & $5,066(50.2)$ \\
\hline Female & $1,116(52.0)$ & $1,888(49.1)$ & $2,018(49.3)$ & $5,022(49.8)$ \\
\hline \multicolumn{5}{|l|}{ Ethnicity, $n(\%)$} \\
\hline Caucasian & $1,381(69.1)$ & $2,356(64.1)$ & $2,818(73.6)$ & $6,555(69.0)$ \\
\hline Asian & $405(20.3)$ & $738(20.1)$ & 715 (18.7) & $1,858(19.5)$ \\
\hline Black & $51(2.6)$ & $259(7.0)$ & $141(3.7)$ & $451(4.7)$ \\
\hline Multiracial & $10(0.5)$ & $16(0.4)$ & $30(0.8)$ & $56(0.6)$ \\
\hline Other/unknown & $298(13.9)$ & $477(12.4)$ & $393(9.6)$ & $1,168(11.6)$ \\
\hline \multicolumn{5}{|l|}{ Age, years } \\
\hline Mean (SD) & $56.1(17.0)$ & $58.1(17.1)$ & $60.4(16.4)$ & $58.6(16.9)$ \\
\hline Median (IQR) & $57.6(43.0-68.8)$ & $59.6(45.6-71.6)$ & $62.7(49.1-73.1)$ & $60.5(46.4-71.8)$ \\
\hline Min-max & $18.5-100.1$ & $18.0-96.7$ & $17.8-96.0$ & $17.8-100.1$ \\
\hline \multicolumn{5}{|l|}{ Age group, $n(\%)$} \\
\hline$<50$ & $787(36.7)$ & $1,247(32.4)$ & $1,068(26.1)$ & $3,102(30.7)$ \\
\hline $50-<65$ & $626(29.2)$ & $1,143(29.7)$ & $1,190(29.0)$ & 2,959 (29.3) \\
\hline $65-<75$ & $438(20.4)$ & 751 (19.5) & $987(24.1)$ & $2,176(21.6)$ \\
\hline $75-<85$ & $232(10.8)$ & $546(14.2)$ & $687(16.8)$ & $1,465(14.5)$ \\
\hline$>85$ & $62(2.9)$ & $159(4.1)$ & $165(4.0)$ & $386(3.8)$ \\
\hline \multicolumn{5}{|l|}{ BMI, $\mathrm{kg} / \mathrm{m}^{2}$} \\
\hline Mean (SD) & $28.1(6.2)$ & $28.0(6.4)$ & $28.9(6.9)$ & $28.4(6.6)$ \\
\hline Median (IQR) & $27.2(24.0-30.9)$ & $27.2(23.9-31.1)$ & $27.7(24.3-32.1)$ & $27.4(24.1-31.5)$ \\
\hline Min-max & $14.2-68.2$ & $12.5-72.3$ & $14.0-86.5$ & $12.5-86.5$ \\
\hline \multicolumn{5}{|l|}{ Care setting, $n(\%)$} \\
\hline Hospital & $1,300(60.6)$ & $2,643(68.7)$ & $3,430(83.7)$ & $7,373(73.1)$ \\
\hline Outpatient & $845(39.4)$ & $1,203(31.3)$ & $667(16.3)$ & $2,715(26.9)$ \\
\hline \multicolumn{5}{|l|}{ Speciality } \\
\hline Vascular medicine & 1,279 (59.6) & $2,016(52.4)$ & $1,194(29.2)$ & $4,489(44.5)$ \\
\hline Internal medicine & $646(30.1)$ & $1,479(38.5)$ & $2,293(56.0)$ & $4,418(43.8)$ \\
\hline General practitioner & $75(3.5)$ & $122(3.2)$ & $179(4.4)$ & $376(3.7)$ \\
\hline Emergency medicine & $59(2.8)$ & $85(2.2)$ & $110(2.7)$ & $254(2.5)$ \\
\hline Cardiology & $86(4.0)$ & $143(3.7)$ & $318(7.8)$ & $547(5.4)$ \\
\hline
\end{tabular}

Abbreviations: BMI, body mass index; IDDVT, isolated distal deep vein thrombosis; IQR, interquartile range; PDVT, proximal deep vein thrombosis; $\mathrm{PE}$, pulmonary embolism; SD, standard deviation.

of IDDVT, PDVT, and PE are presented in - Supplementary Table S1 (available in the online version). The ratio between the incidence of IDDVT to that of PDVT varied strikingly across countries, ranging from 0.15 (95\% CI, 0.11-0.18) in Canada to $1.96(95 \% \mathrm{CI}, 1.51-2.41)$ in Australia (-Supplementary Fig. S1, available in the online version).

Most patients (> 60\%) with IDDVT, PDVT, and PE had at least one identifiable provoking risk factor within 3 months prediagnosis (-Fig. 2). Specifically, IDDVT patients were more likely to have undergone surgery (14.6\%) or experienced trauma to a lower limb (13.2\%) than PDVT patients (11.0 and
8.7\%, respectively) and PE patients ( 12.7 and $4.5 \%$, respectively). Compared with IDDVT, patients with PDVT or PE were more likely to have active cancer (7.2\% vs. $9.9 \%$ and $10.3 \%)$ and a prior history of VTE (13.8\% vs. $17.2 \%$ and $14.6 \%$ ) (-Fig. 2 ).

Pretest probability scores (e.g., Wells score) were used in only $4.9 \%$ of the overall sample. DVT was diagnosed using CUS in nearly all cases (95.8\%) with a positive D-dimer assay obtained in approximately one-quarter (26.3\%). Contrast venography was rarely used (1.3\%). PE was diagnosed by computed tomography (CT) pulmonary angiography in $91.8 \%$ of patients. 


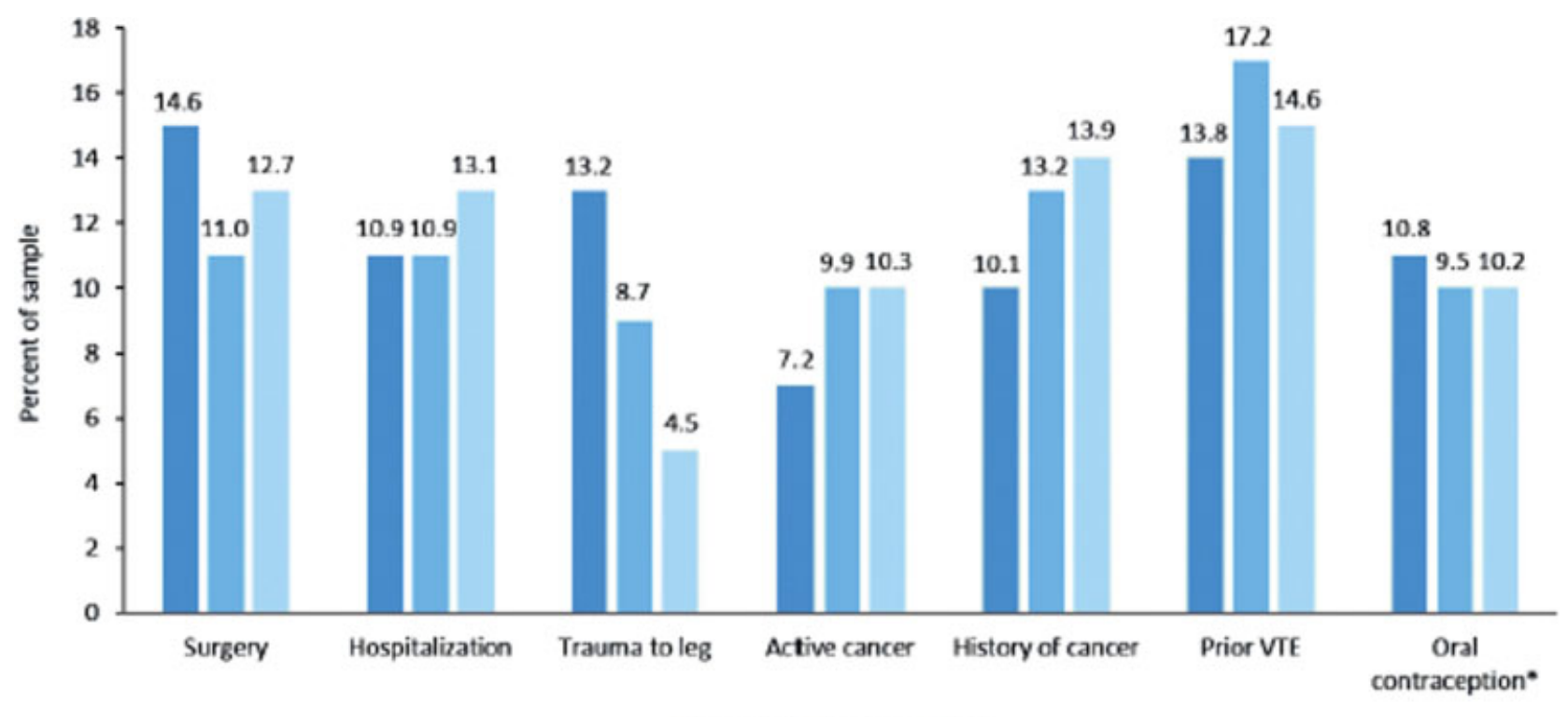

IDDVT $=\mathrm{PDVT}=\mathrm{PE}$

Fig. 2 Provoking risk factors in patients with isolated distal deep vein thrombosis (IDDVT), proximal DVT (PDVT) \pm distal, or pulmonary embolism (PE) \pm DVT within the previous 3 months prior to diagnosis. Most frequently encountered provoking factors arising in more than $5 \%$ IDDVT group are shown. Individual patients could have $\geq 1$ provoking factor. ${ }^{*}$ Counted as a percentage of women.

\section{Treatment}

A lower proportion of patients with IDDVT was treated as inpatients compared with those with PDVT and PE (60.6\% vs. $68.7 \%$ and $83.7 \%$, respectively). All nonhospitalized patients were classified as outpatients.

Following diagnosis, nearly all patients (IDDVT, PDVT, and PE) were initiated on anticoagulant therapy either alone (overall, 93.0\%) or in combination with other treatments such as thrombolytics (- Table 2). In all three subgroups, treatment started with parenteral anticoagulant plus vitamin K antagonist
(VKA) or DOAC alone more often than with parenteral anticoagulant alone (overall, $59.1 \%$ vs. $16.2 \%$ ). Patients with IDDVT were more likely to receive DOAC alone as initial therapy compared with those with PDVT and PE (44.5\% vs. 32.3\% and 26.4\%). Overall, use of graded compression stockings (GCS) was 35.7\%. Patients with IDDVT or PDVT were more likely to receive GCS than those with PE ( $42.8 \%$ and $46.9 \%$ vs. $21.5 \%$ ). Only $2.1 \%$ patients received no therapy or GCS only (-Table 2 ).

The duration of anticoagulant treatments is shown in -Fig. 3. Extension of anticoagulant therapy beyond 3 months

Table 2 Initial treatment provided ( $\leq 30$ days postdiagnosis)

\begin{tabular}{|c|c|c|c|c|}
\hline & $\begin{array}{l}\text { IDDVT } \\
(N=2,145)\end{array}$ & $\begin{array}{l}\text { PDVT } \\
(N=3,846)\end{array}$ & $\begin{array}{l}\mathrm{PE} \\
(N=4,097)\end{array}$ & $\begin{array}{l}\text { Total } \\
(N=10,088)\end{array}$ \\
\hline AC only & $2,012(93.8)$ & $3,583(93.2)$ & $3,791(92.5)$ & $9,386(93.0)$ \\
\hline Parenteral therapy only & $348(17.3)$ & $579(16.2)$ & $593(15.6)$ & $1,520(16.2)$ \\
\hline Parenteral therapy + VKA & $385(19.1)$ & $1,112(31.0)$ & $1,002(26.4)$ & $2,499(26.6)$ \\
\hline VKA only & $137(6.8)$ & $218(6.1)$ & $210(5.5)$ & $565(6.0)$ \\
\hline DOAC only & $896(44.5)$ & $1,158(32.3)$ & $1,001(26.4)$ & $3,055(32.5)$ \\
\hline Parenteral therapy + DOAC & $185(9.2)$ & $440(12.3)$ & $904(23.8)$ & $1,529(16.3)$ \\
\hline Other AC & $21(1.0)$ & $32(0.9)$ & $37(1.0)$ & $90(1.0)$ \\
\hline Other therapy $+\mathrm{AC}$ & $69(3.2)$ & $182(4.7)$ & $239(5.8)$ & $490(4.9)$ \\
\hline Thrombolytic therapy $+\mathrm{AC}$ & $35(1.6)$ & $97(2.5)$ & $160(3.9)$ & $292(2.9)$ \\
\hline Surgical or mechanical $+A C$ & $23(1.1)$ & $49(1.3)$ & $52(1.3)$ & $124(1.2)$ \\
\hline GCS & $919(42.8)$ & $1,803(46.9)$ & $882(21.5)$ & $3,604(35.7)$ \\
\hline No therapy or GCS only & $64(3.0)$ & $81(2.1)$ & $67(1.6)$ & $212(2.1)$ \\
\hline
\end{tabular}

Abbreviations: AC, anticoagulant; DOAC, direct oral anticoagulant; GCS, graduated compression stocking; IDDVT, isolated distal deep vein thrombosis; PDVT, proximal deep vein thrombosis; PE, pulmonary embolism; VKA, vitamin K antagonist. 
was less likely to occur in patients with IDDVT than in those with PDVT or PE. In the IDDVT, PDVT, and PE groups, the proportion of patients receiving anticoagulant therapy was 61.7, 73.9, and $81.0 \%$ at 6 months and $45.8,54.7$, and $61.9 \%$ at 12 months. At 3, 6, and 12 months after diagnosis, IDDVT patients who were receiving anticoagulant therapy were being treated with VKA in approximately one-quarter cases and DOAC in approximately half of the cases at all three time points. Among patients with PDVT and PE, those who were receiving anticoagulant therapy were being treated with VKA and DOAC in one-third and one-half cases, respectively, at all three time points. The majority of patients on anticoagulant therapy who were not being treated with either VKA only or DOAC only were receiving a parenteral anticoagulant at 3,6 , and 12 months postdiagnosis (-Fig. 3 ).

\section{Clinical Outcomes}

One-year outcomes calculated per 100 person-years are displayed in - Table 3. In the IDDVT, PDVT, and PE groups, VTE recurrence rates and their associated 95\% CI were 4.8 (3.9-5.9), 6.5 (5.7-7.4), and 4.2 (3.6-4.9)/100 person-years, respectively, whereas all-cause mortality rates were 4.6 (3.8-5.7), 8.0 (7.1-9.0), and 7.7 (6.8-8.6)/100 person-years, respectively. Incidence of newly diagnosed cancer was 1.3 (0.9-2.0), 2.5 (2.0-3.1), and $2.6(2.1-3.2) / 100$ person-years, respectively.

A forest plot of sHRs for predefined adverse outcomes in patients with IDDVT versus PDVT is provided in - Fig. 4. Over 12 months of follow-up, the incidence of all-cause mortality and de novo cancer was significantly lower in IDDVT patients than PDVT patients (HR, 0.61 [95\% CI, 0.48-0.77]; $p<0.0001$ and sHR, 0.60 [95\% CI, 0.39-0.93]; $p=0.0229$, respectively). Incidence rate for recurrent VTE was significantly lower for IDDVT than PDVT patients (sHR, 0.76 [95\% CI, 0.60-0.97]; $p=0.0298$ ). IDDVT patients were significantly less likely to experience bleeding events (major or otherwise) (sHR, 0.69 [95\% CI, 0.57-0.84]; $p=0.0002$ ), although the incidence of major bleeding was not significantly different between groups (sHR, 0.80 [95\% CI, 0.49-1.28]; $p=0.3503$ ). The incidence of arterial thrombotic events was not significantly different between patients with IDDVT and PDVT (-Fig. 4).

Differences in the relative risk of experiencing a range of adverse outcomes during 12 months postdiagnosis were even more pronounced in patients with IDDVT versus PE; outcomes of interest are shown in -Fig. 5. Apart from recurrent VTE (sHR, 1.16 [95\% CI, 0.89-1.51]; $p=0.2663$ ) and stroke/TIA (sHR, 0.59 [95\% CI, 0.29-1.20]; $p=0.1458$ ), all adverse outcomes including death and cancer were significantly (all $p<0.05$ ) less likely to occur in patients with IDDVT than in those with PE.

\section{Influence of Provoking Factors on Future Risk}

Results of investigation into whether location of leg DVT interacts with recent provoking factors to influence risk are presented in - Table $\mathbf{4}$ and - Fig. 6. In patients with unprovoked DVT and those with active cancer, risk of all adverse outcomes was generally lower in the IDDVT versus PDVT group albeit nonsignificantly. On the other hand, in patients

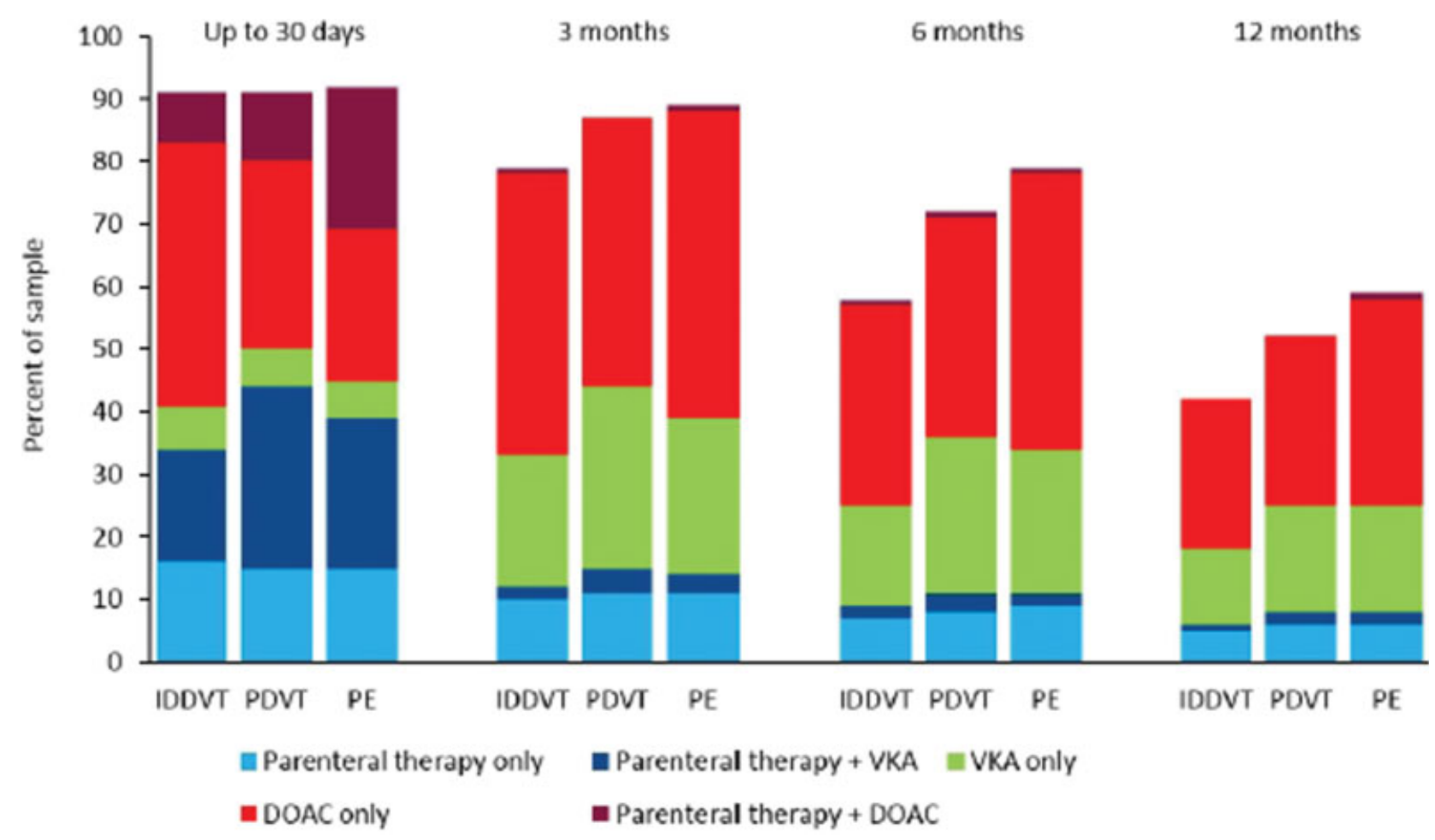

Fig. 3 Time course of anticoagulation strategies. Over 1 year, persistence with anticoagulant therapy gradually tapered off in all three groups and especially in the isolated distal deep vein thrombosis (IDDVT) group. Most patients were started on either parenteral therapy \pm vitamin $\mathrm{K}$ antagonist (VKA) or direct oral anticoagulant (DOAC). Thereafter, at months 3, 6, and 12 the majority of patients still taking anticoagulant therapy were maintained on DOAC. 
Table 3 One-year outcomes, rate per 100 person-years $(95 \% \mathrm{Cl})$

\begin{tabular}{|l|l|l|l|l|}
\hline & $\begin{array}{l}\text { IDDVT } \\
(N=2,123)\end{array}$ & $\begin{array}{l}\text { PDVT } \\
(N=3,830)\end{array}$ & $\begin{array}{l}\text { PE } \\
(N=4,066)\end{array}$ & $\begin{array}{l}\text { Total } \\
(N=10,019)\end{array}$ \\
\hline Death (all causes) & $4.6(3.8$ to 5.7$)$ & $8.0(7.1$ to 9.0$)$ & $7.7(6.8$ to 8.6$)$ & $7.2(6.6$ to 7.7$)$ \\
\hline Bleeding, any & $7.5(6.3$ to 8.8$)$ & $10.9(9.8$ to 12.1$)$ & $12.8(11.6$ to 14.0$)$ & $10.9(10.2$ to 11.6$)$ \\
\hline Bleeding, major & $1.2(0.8$ to 1.8$)$ & $1.6(1.2$ to 2.1$)$ & $2.4(1.9$ to 2.9$)$ & $1.8(1.6$ to 2.1$)$ \\
\hline Cancer & $1.3(0.9$ to 2.0$)$ & $2.5(2.0$ to 3.1$)$ & $2.6(2.1$ to 3.2$)$ & $2.3(2.0$ to 2.6$)$ \\
\hline MI/ACS & $0.3(0.1$ to 0.7$)$ & $0.7(0.5$ to 1.0$)$ & $0.9(0.7$ to 1.3$)$ & $0.7(0.5$ to 0.9$)$ \\
\hline Recurrent VTE episode & $4.8(3.9$ to 5.9$)$ & $6.5(5.7$ to 7.4$)$ & $4.2(3.6$ to 4.9$)$ & $5.2(4.8$ to 5.7$)$ \\
\hline Stroke/TIA & $0.5(0.3$ to 1.0$)$ & $0.6(0.4$ to 1.0$)$ & $1.0(0.8$ to 1.4$)$ & $0.8(0.6$ to 1.0$)$ \\
\hline
\end{tabular}

Abbreviations: ACS, acute coronary syndrome; $\mathrm{Cl}$, confidence interval; IDDVT, isolated distal deep vein thrombosis; MI, myocardial infarction; PDVT, proximal deep vein thrombosis; PE, pulmonary embolism; TIA, transient ischemic attack; VTE, venous thromboembolism.

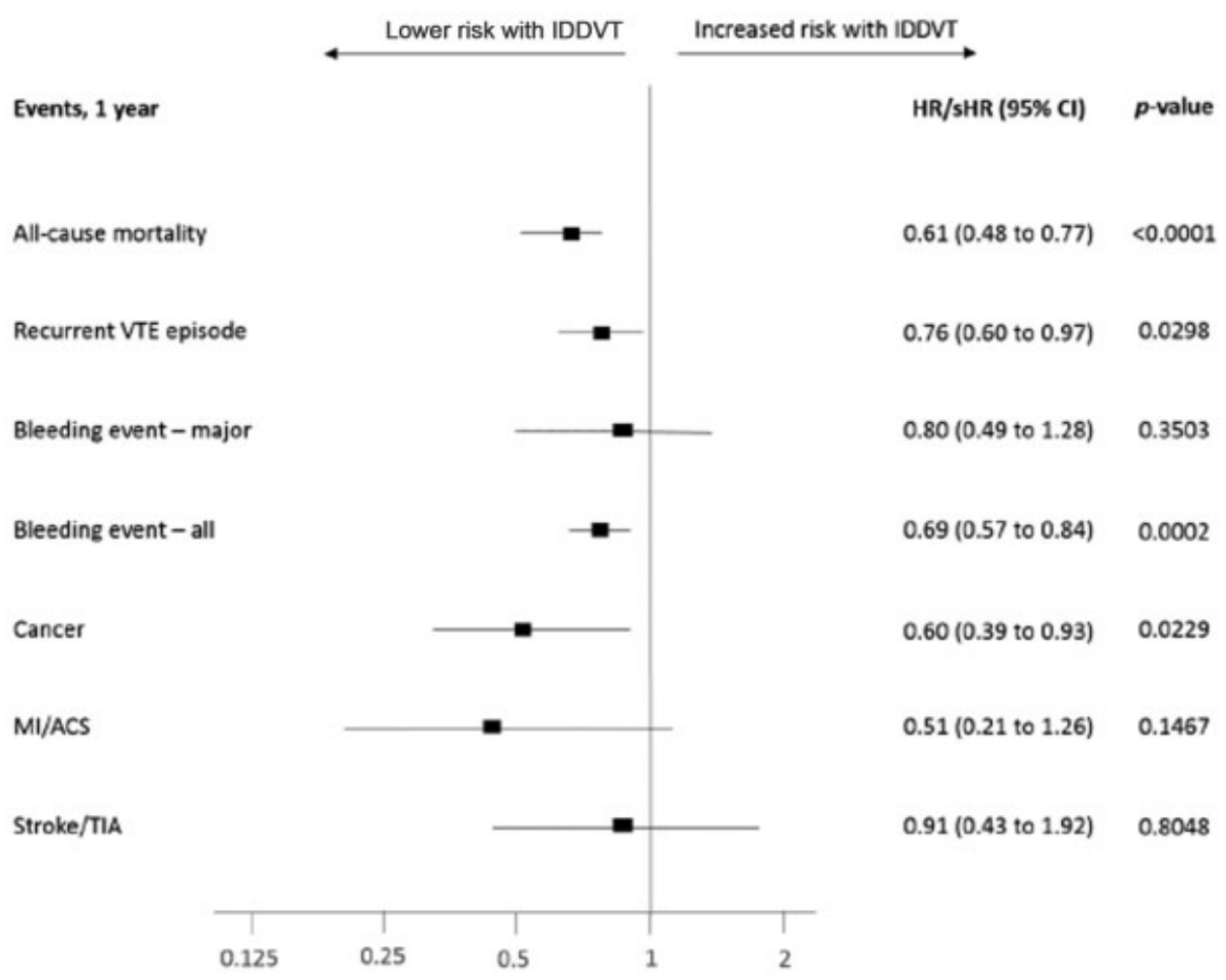

Fig. 4 Outcomes in isolated distal deep vein thrombosis (IDDVT) versus proximal DVT (PDVT) (reference) group, days 0 to 365 . Hazard ratio (HR) for death and subhazard ratios (sHRs) for other outcomes with death as competing risk.

with transient provoking factors risk of all-cause mortality and recurrence was significantly lower in IDDVT versus PDVT patients and hazard for major bleeding somewhat lower albeit nonsignificantly.

\section{Discussion}

This study investigated the clinical characteristics, treatment patterns, and 1-year outcomes in a total of 10,685 patients with IDDVT, PDVT, and PE using real-world data as captured by the global GARFIELD-VTE registry. ${ }^{16}$ The data set included 2,145 patients with IDDVT (21.3\%), 3,846 with PDVT (38.1\%), and 4,097 with PE (40.6\%), mostly from Europe.

The present analysis both confirms what has been previously demonstrated in this clinical context and sheds new light on some aspects of the disease. In line with previous estimates, 6,21,22 IDDVT patients accounted for approximately one-third of all patients with lower limb DVT $(2,145$ of 5,991 patients overall). Based on the observed frequencies, there was a trend toward a higher proportion of IDDVT patients having recent provoking factors compared with those with PDVT or PE. PDVT and PE were more closely associated with an underlying disease condition (especially cancer) or previous episode of VTE. Diagnosis and treatment patterns suggest that the GARFIELD-VTE cohort largely received standard care according to international guidelines-with some notable exceptions (see below). DVT was diagnosed using CUS in nearly all cases with a positive D-dimer assay frequently obtained, although a pretest probability score (e.g., Wells score) was rarely 




Fig. 5 Outcomes in isolated distal deep vein thrombosis (IDDVT) versus pulmonary embolism (PE) (reference) group, days 0 to 365 . Hazard ratio (HR) for death and subhazard ratios (sHRs) for other outcomes with death as competing risk.

Table 4 Major outcomes in IDDVT versus PDVT patients stratified by the presence of transient provoking factors, persistent risk factor (active cancer), or no provoking factor, rate per 100 person-years $(95 \% \mathrm{Cl})$

\begin{tabular}{|l|l|l|}
\hline Risk group/outcome & $\begin{array}{l}\text { IDDVT } \\
(\boldsymbol{n}=\mathbf{2 , 1 2 4 )}\end{array}$ & $\begin{array}{l}\text { PDVT } \\
\text { ( } \boldsymbol{n}=3,831)\end{array}$ \\
\hline Active cancer & & \\
\hline All-cause mortality & $40.0(29.9$ to 53.4$)$ & $55.3(46.9$ to 65.1$)$ \\
\hline Recurrent VTE episode & $11.9(6.9$ to 20.4$)$ & $12.8(9.0$ to 18.1$)$ \\
\hline Bleeding event-major & $8.0(4.2$ to 15.3$)$ & $5.5(3.2$ to 9.3$)$ \\
\hline Transient provoking factor & & \\
\hline All-cause mortality & $1.6(0.9$ to 2.8$)$ & $5.8(4.5$ to 7.4$)$ \\
\hline Recurrent VTE episode & $3.0(2.0$ to 4.6$)$ & $6.4(5.0$ to 8.2$)$ \\
\hline Bleeding event-major & $1.1(0.5$ to 2.2$)$ & $1.7(1.1$ to 2.7$)$ \\
\hline Unprovoked & & \\
\hline All-cause mortality & $3.0(2.1$ to 4.2$)$ & $3.5(2.8$ to 4.4$)$ \\
\hline Recurrent VTE episode & $5.4(4.1$ to 6.9$)$ & $5.8(4.8$ to 6.9$)$ \\
\hline Bleeding event-major & $0.6(0.3$ to 1.3$)$ & $1.1(0.7$ to 1.6$)$ \\
\hline
\end{tabular}

Abbreviations: $\mathrm{Cl}$, confidence interval; IDDVT, isolated distal deep vein thrombosis; PDVT, proximal deep vein thrombosis; VTE, venous thromboembolism.

used. The diagnosis of PE was usually confirmed by CT pulmonary angiography.

Overall, IDDVT patients comprised approximately onethird of the entire cohort of DVT. However, the relative proportions of IDDVT versus PDVT varied considerably from country to country, with some centers paradoxically showing a marked preponderance of the former over latter presentation. Rather than being a reflection of the true epidemiologic picture, or an artifact of random sampling, this finding could be due to different local practices in performing diagnostic tests. It is believed that many centers worldwide limit CUS scan to proximal veins while others perform whole-leg imaging. ${ }^{12}$ Furthermore, there is no uniform definition for "IDDVT," 5 and it is conceivable that 


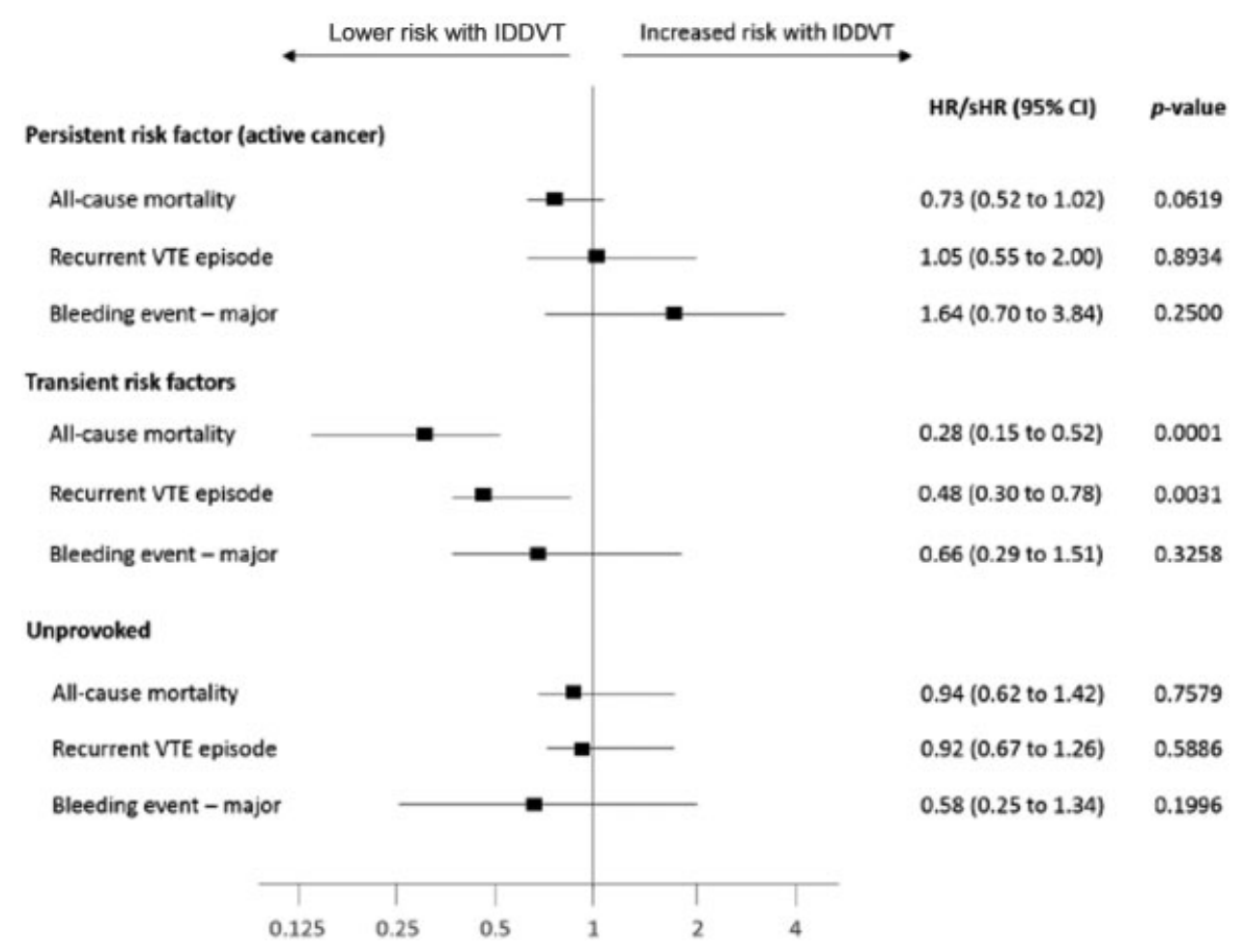

Fig. 6 Outcomes in isolated distal deep vein thrombosis (IDDVT) versus proximal DVT (PDVT) patients stratified by the presence of persistent provoking factor (active cancer), transient provoking factors (at least one of surgery, hospitalization, pregnancy, hormone replacement therapy/ oral contraception, acute medical illness, or trauma of lower limb within 3 months prior to enrolment), or no provoking factor.

some patients with thrombosis involving calf muscle veins and not axial vessels might have been excluded. These observations cast doubts in two directions. In some countries there might exist underdiagnosis, with potential harm due to missed cases, whereas elsewhere overdiagnosis due to false positives or picking up clinically irrelevant cases may cause potential harm due to unnecessary treatment, as other authors have also suggested. ${ }^{11}$

Although treatment patterns in the GARFIELD-VTE cohort appear to follow guideline-recommended practice, these real-world data reveal some interesting divergences. Notably, nearly all patients with IDDVT received anticoagulant therapy, including chronic anticoagulant therapy. Two recent, large-scale clinical investigations ${ }^{12,23}$ have suggested that anticoagulant therapy had little utility and was possibly harmful in low-risk patients (the majority) with IDDVT. International clinical practice guidelines ${ }^{13,14}$ reflect these results by not generally advocating anticoagulant therapy against IDDVT, unless severe, and instead recommend risk stratification in these patients. In the present study, only $13.8 \%$ had prior VTE and $7.2 \%$ active cancer. On the other hand, nearly half reported recent provoking factors within 30 days prior to enrolment. This suggests that the majority would be considered "low risk" in whom anticoagulant therapy is not generally recommended. However, active treatment of emergent IDDVT rather than risk-stratified therapy appears routine practice globally.

Decision to extend anticoagulant therapy beyond 3 months was widespread, including in patients with IDDVT. This finding is surprising for at least two reasons: recurrence rate is believed lower in patients with IDDVT than in those with PDVT, ${ }^{24,25}$ and this disease entity is often caused by temporary provoking factors, ${ }^{21,22}$ as the present study appears to confirm. Use of prolonged ( $>3$ months) anticoagulant therapy was even higher in patients with PDVT and PE. For both initial treatment and longer-term anticoagulation, DOACs were more frequently selected than VKA in all VTE patients. As a corollary of the high proportion of patients who received extensive anticoagulation therapy, there was a very low prevalence of IDDVT patients who received no anticoagulation or GCS (3.0\%), despite guidelines (e.g., $\mathrm{ACCP}^{13,14}$ ) recommending this strategy in many low-risk individuals.

Some recent evidence suggests that the prognostic significance of leg DVT location (IDDVT or PDVT) interacts with the presence of provoking factors, especially cancer. ${ }^{24-26}$ Valerio's group $^{26}$ showed that in the absence of provoking factors patients with IDDVT were less likely to experience recurrence than those with PDVT, whereas the prognostic impact of DVT location was weaker if the lesion were provoked. In contrast, the present study shows that the risk of recurrence and death was decreased in IDDVT versus PDVT patients with transient provoking factors, and that DVT location was a less important prognostic indicator in unprovoked patients and those with cancer. Hence, controversy remains as to whether the presence of transient provoking factors influences future risk or the decision to treat IDDVT.

This study has a number of limitations. Apart from its realworld registry design (unselected, consecutive patients), there was no control group of IDDVT patients with which to compare treatment patterns and their success. For example, recurrence 
rates in patients who continued long-term anticoagulant therapy versus those who stopped treatment early are unknown, as are recurrence rates in untreated patients with IDDVT versus PDVT. As noted above, specific site of IDDVT was not recorded (axial or muscular), which may have impacted treatment strategy.

In conclusion, the GARFIELD-VTE registry provides realworld data showing how patients with VTE are being diagnosed and treated around the world. This study suggests that patients presenting with IDDVT are likely to have experienced recent provoking factors such as leg trauma and hospitalization. They are less likely to develop cancer compared with PDVT and PE patients, and have a lower HR for experiencing VTE recurrence. Almost all IDDVT patients received anticoagulant therapies, in nearly half of the cases for at least 12 months. Very few IDDVT patients were deferred anticoagulants. These findings point to a need for better-defined risk stratification for IDDVT as well as closer implementation of guideline-recommended practice.

\section{What is known about this topic?}

- In many cases isolated distal DVT (IDDVT) occurring below the knee may spontaneously resolve; on the other hand, it could extend proximally and lead to pulmonary embolism.

- Decision whether to provide anticoagulation against IDDVT is influenced by perceived level of risk.

\section{What does this paper add?}

- In this large, global, observational cohort study patterns of diagnosis and treatment of IDDVT were analyzed and compared with proximal DVT (PDVT) and pulmonary embolism (PE).

- IDDVT was more often associated with transient provoking factors whereas PDVT and PE likelier resulted from persistent provoking factors.

- Risk of serious outcomes such as death and cancer was lower for IDDVT than for PDVT and PE.

- All patients with IDDVT, PDVT, and PE received anticoagulation therapy.

- Controversy remains as to whether the presence of provoking factors predicts future risk in patients with IDDVT.

\section{Funding}

The GARFIELD-VTE registry is an independent academic research initiative sponsored by the Thrombosis Research Institute (London, UK) and supported by an unrestricted research grant from Bayer Pharma AG (Berlin, Germany).

\section{Conflict of Interest}

S.S.: Speaker fees from Bayer Healthcare, Boehringer Ingelheim, Bristol-Myers Squibb, Daiichi-Sankyo, Sanofi
Aventis, and Pfizer; consultancy fees from Bayer Healthcare, Boehringer Ingelheim, Daiichi-Sankyo, Sanofi Aventis, Pfizer. S.Z.G.: Grants from BiO2 Medical, BoehringerIngelheim, Bristol-Myers Squibb, BTG EKOS, Daiichi-Sankyo, National Heart Lung and Blood Institute of the National Institutes of Health, Janssen, Thrombosis Research Group; personal fees (all consultancy less than $\$ 10 \mathrm{k}$ ) from Bayer, Boehringer-Ingelheim, Bristol-Myers Squibb, Daiichi-Sankyo, Janssen, Portola, Zafgen. J.I.W.: Honoraria from Bayer, Boehringer Ingelheim, BristolMyers Squibb, Daiichi-Sankyo, Ionis, Janssen, Merck, Portola, Pfizer, Servier, Novartis. W.A.: Grants and personal fees from Bayer. Personal fees from Boehringer Ingelheim, Daiichi-Sankyo, BMS-Pfizer, Sanofi, Portola, Aspen, Stago, CSL Behring. H.B.: Grants and personal fees from Thrombosis Research Institute, London. Personal fees from Bayer Switzerland. A.G.G.T.: Personal fees from Bayer Pharma AG, Janssen. P.A.: None declared. S.H.: Honoraria from Aspen, Bayer Healthcare, BMS, Daiichi-Sankyo, Pfizer, Portola, Sanofi. S.G.: Research grant from Ono, BristolMyers Squibb, Sanofi, Pfizer. A.Z.: None declared. A.F.: None declared. J.D.N.: Honoraria from Bayer, BoehringerIngelheim, Bristol-Myers Squibb, Leo Pharma, Pfizer. G.K.: None declared. L.G.M.: Grants and personal fees from Bayer Healthcare, Boehringer Ingelheim, Pfizer, DaiichiSankyo. P.P.: Personal fees from Bayer Pharma, Pfizer, Daiichi-Sankyo, Sanofi, Rovi Pharmaceuticos. A.K.K.: Research grants from Bayer Healthcare. Personal fees from Bayer Healthcare, Boehringer-Ingelheim Pharma, DaiichiSankyo Europe, Sanofi SA, Janssen Pharma, Verseon Inc., Pfizer.

\section{Acknowledgments}

The authors thank the physicians, nurses, and patients involved in the GARFIELD registry. Alex Kahney of Thrombosis Research Institute (TRI) provided medical writing support. Henrik Fryk of TRI performed some of the statistical analyses. Madhusudana Rao of TRI was SAS programmer.

\section{References}

1 Piazza G, Goldhaber SZ. Physician alerts to prevent venous thromboembolism. J Thromb Thrombolysis 2010;30(01):1-6

2 Stevens SM, Douketis JD. Deep vein thrombosis prophylaxis in hospitalized medical patients: current recommendations, general rates of implementation, and initiatives for improvement. Clin Chest Med 2010;31(04):675-689

3 Wendelboe AM, Raskob GE. Global burden of thrombosis-epidemiologic aspects. Circ Res 2016;118(09):1340-1347

4 ISTH Steering Committee for World Thrombosis Day. Thrombosis: a major contributor to the global disease burden. J Thromb Haemost 2014;12(10):1580-1590

5 Palareti G, Schellong S. Isolated distal deep vein thrombosis: what we know and what we are doing.J Thromb Haemost 2012;10(01): $11-19$

6 Robert-Ebadi H, Righini M. Management of distal deep vein thrombosis. Thromb Res 2017;149:48-55

7 Franco L, Giustozzi M, Agnelli G, Becattini C. Anticoagulation in patients with isolated distal deep vein thrombosis: a metaanalysis. J Thromb Haemost 2017;15(06):1142-1154 
8 Horner D, Hogg K, Body R, Nash MJ, Baglin T, Mackway-Jones K. The anticoagulation of calf thrombosis (ACT) project: results from the randomized controlled external pilot trial. Chest 2014;146 (06):1468-1477

9 Donadini MP, Dentali F, Pegoraro S, et al. Long-term recurrence of venous thromboembolism after short-term treatment of symptomatic isolated distal deep vein thrombosis: a cohort study. Vasc Med 2017;22(06):518-524

10 Kearon C. Natural history of venous thromboembolism. Circulation 2003;107(23, Suppl 1):I22-I30

11 Righini M, Paris S, Le Gal G, Laroche JP, Perrier A, Bounameaux H. Clinical relevance of distal deep vein thrombosis. Review of literature data. Thromb Haemost 2006;95(01):56-64

12 Righini M, Galanaud J-P, Guenneguez H, et al. Anticoagulant therapy for symptomatic calf deep vein thrombosis (CACTUS): a randomised, double-blind, placebo-controlled trial. Lancet Haematol 2016; 3(12):e556-e562

13 Kearon C, Akl EA, Comerota AJ, et al. Antithrombotic therapy for VTE disease: Antithrombotic Therapy and Prevention of Thrombosis, 9th ed.: American College of Chest Physicians Evidence-Based Clinical Practice Guidelines. Chest 2012;141(2, Suppl):e419S-e496S

14 Kearon C, Akl EA, Ornelas J, et al. Antithrombotic therapy for VTE disease: CHEST guideline and expert panel report. Chest 2016; 149(02):315-352

15 Blann AD, Khoo CW. The prevention and treatment of venous thromboembolism with LMWHs and new anticoagulants. Vasc Health Risk Manag 2009;5:693-704

16 Weitz JI, Haas S, Ageno W, et al. Global Anticoagulant Registry in the Field - Venous Thromboembolism (GARFIELD-VTE). Rationale and design. Thromb Haemost 2016;116(06):1172-1179

17 Schulman S, Kearon C; Subcommittee on Control of Anticoagulation of the Scientific and Standardization Committee of the International Society on Thrombosis and Haemostasis. Definition of major bleeding in clinical investigations of antihemostatic medicinal products in non-surgical patients. J Thromb Haemost 2005;3(04):692-694
18 Kearon C, Ageno W, Cannegieter SC, Cosmi B, Geersing GJ, Kyrle PA; Subcommittees on Control of Anticoagulation, and Predictive and Diagnostic Variables in Thrombotic Disease. Categorization of patients as having provoked or unprovoked venous thromboembolism: guidance from the SSC of ISTH. J Thromb Haemost 2016; 14(07):1480-1483

19 Fine JP, Gray RJ. A proportional hazards model for the subdistribution of a competing risk. J Am Stat Assoc 1999;94(446): 496-509

20 Ageno W, Haas S, Weitz JI, et al; GARFIELD-VTE investigators. Characteristics and management of patients with venous thromboembolism: the GARFIELD-VTE registry. Thromb Haemost 2019; 119(02):319-327

21 Galanaud JP, Sevestre-Pietri MA, Bosson JL, et al; OPTIMEV-SFMV Investigators. Comparative study on risk factors and early outcome of symptomatic distal versus proximal deep vein thrombosis: results from the OPTIMEV study. Thromb Haemost 2009;102(03): 493-500

22 Galanaud JP, Quenet S, Rivron-Guillot K, et al; RIETE INVESTIGATORS. Comparison of the clinical history of symptomatic isolated distal deep-vein thrombosis vs. proximal deep vein thrombosis in 11086 patients. J Thromb Haemost 2009;7(12):2028-2034

23 Utter GH, Dhillon TS, Salcedo ES, et al. Therapeutic anticoagulation for isolated calf deep vein thrombosis. JAMA Surg 2016;151 (09): 161770

24 Galanaud JP, Sevestre MA, Genty C, et al; OPTIMEV-SFMV investigators. Incidence and predictors of venous thromboembolism recurrence after a first isolated distal deep vein thrombosis. J Thromb Haemost 2014;12(04):436-443

25 Barco S, Corti M, Trinchero A, et al. Survival and recurrent venous thromboembolism in patients with first proximal or isolated distal deep vein thrombosis and no pulmonary embolism. J Thromb Haemost 2017;15(07):1436-1442

26 Valerio L, Ambaglio C, Barone M, et al. Recurrence risk after first symptomatic distal versus proximal deep vein thrombosis according to baseline risk factors. TH Open 2019;3(01):e58-e63 